\title{
A model for client recommendation to a desktop grid server
}

\begin{abstract}
A vast amount of idle computational power of desktop computers could be utilized throughout desktop grids. For an appropriate utilization, the scheduler, needs to determine clients which are best suited to deliver assigned jobs in time. Diversity of hosts (i.e. OS, hardware and network specifications) and intermittent availability of resources are known issues which complicate the schedulers work. As a solution to this problem, a clientï server model consisting two modules for a desktop grid middleware is discussed: a module to forecast machine resource availability in the client side and a module in the server side that recommends clients to the scheduler that are the nearest to job expectations. Historic data, time-series analyses and machine learning are used for this purpose in the modules.
\end{abstract}

Keyword: Desktop grid; Availability prediction; Case-based reasoning; Match-making; Recommender system 